\title{
The Safety of Fecal Microbiota Transplantation for Crohn's Disease: Findings from A Long-Term Study
}

\author{
Honggang Wang · Bota Cui • Qianqian Li · Xiao Ding · \\ Pan Li · Ting Zhang · Xiaozhong Yang · Guozhong Ji • \\ Faming Zhang (D)
}

Received: August 18, 2018 / Published online: October 16, 2018

(C) The Author(s) 2018

\section{ABSTRACT}

Introduction: Fecal microbiota transplantation (FMT) has been used as a potential treatment option for Crohn's disease (CD). However, there is still lack of safety and efficacy evidence based on large samples of CD undergoing FMT. This

Honggang Wang and Bota Cui contributed equally to this work

Enhanced digital features To view enhanced digital features for this article go to https://doi.org/10.6084/ m9.figshare.7123292.

H. Wang - B. Cui · Q. Li - X. Ding · P. Li .

T. Zhang · G. Ji $(\bowtie) \cdot$ F. Zhang $(\square)$

Medical Center for Digestive Diseases, Second

Affiliated Hospital of Nanjing Medical University,

Nanjing, China

e-mail: jgz@njmu.edu.cn

F. Zhang

e-mail: fzhang@njmu.edu.cn

H. Wang · X. Yang

Department of Gastroenterology, The Affiliated

Huaian No. 1 People's Hospital of Nanjing Medical

University, Huai'an, China

B. Cui · Q. Li - X. Ding - P. Li - T. Zhang - G. Ji .

F. Zhang

Key Lab of Holistic Integrative Enterology, Nanjing

Medical University, Nanjing, China

F. Zhang

National Clinical Research Center for Digestive

Diseases, Xi'an, China study aimed to evaluate the risk factors of adverse event (AE) in the long term and the efficacy of FMT in the short term for patients with CD.

Methods: FMT via mid-gut for mild to severe CD in a single center trial (NCT01793831) was performed from October 2012 to December 2016. The possible factors with AE and efficacy after FMT were prospectively recorded.

Results: A total of 184 frequencies of FMT were performed for 139 patients who received FMT. During 1 month after FMT, $13.6 \%$ of mild AEs occurred, including increased frequency of defecation, fever, abdominal pain, flatulence, hematochezia, vomiturition, bloating and herpes zoster. No AE beyond 1 month was observed. Therefore, a 1 month cut-off could be suggested to define short-term and long-term AEs of FMT. Among the possible risk factors, only fecal microbiota purification methods were closely associated with the occurrence of AEs. The rate of AEs in patients undergoing manual methods for the preparation of fecal microbiota was $21.7 \%$, which was significantly higher than the $8.7 \%$ in those experiencing an automatic method. The manual or automatic purification of fecal microbiota had no correlation with the efficacy of FMT.

Conclusion: This cohort study based on the largest size of cases demonstrated that improved fecal microbiota preparation reduced the rates of AEs, but did not affect the clinical efficacy in patients with $\mathrm{CD}$. 
Keywords: Adverse event; Crohn's disease; Fecal microbiota transplantation; Gastroenterology; Safety

\section{INTRODUCTION}

Dysbiosis has been proven to be associated with intestinal chronic inflammation in Crohn's disease (CD) [1]. Fecal microbiota transplantation (FMT) as a model for reconstructing microbiota has been shown to have a potential role in the treatment of CD [2-7]. With a deep understanding of the gut microbiota, increasing clinical studies of FMT have in recent years been expanding from Clostridium difficile infection (CDI) [8-11] to other diseases mainly in inflammatory bowel diseases (IBD) $[3,6,7,12-16]$. Therefore, the safety of FMT is becoming an important issue in clinical practice. With the consideration of the potential risk in spreading pathogens, the US. Food and Drug Administration has tried to regulate FMT as it investigates new drug and has shifted its position several times. Recently, Hoffmann et al. [17] proposed a three-track regulatory scheme and called for balancing the safety, efficacy, access and research in FMT regulation. Evidence of the safety is the basis for policy making. However, long-term studies on safety based on large sizes has been very limited, and there have been no reports on long-term $\mathrm{AE}$ related to FMT in $\mathrm{CD}$ based on large sample sizes $(>50)$.

We have reported the clinical remission rate of $76.7 \% 1$ month after FMT in 30 CD cases [6]. This result was consistent with the result of the report from Suskind's study [3]. Importantly, most patients with CD need long-term immunosuppressive treatment. Kelly et al. [18] reported that there were no FMT-related infectious complications in immunocompromised patients with CDI. However, it is unknown whether the immunosupression would increase the risk of infection and other AEs after FMT in the long term. Based on our previous studies of single FMT used in refractory CD [6], and the strategy of FMT for CD complicated with inflammatory mass [7], the present study further aimed to evaluate the possible factors involving with short-term and long-term safety of FMT in CD.

\section{METHODS}

\section{Ethics Approval and Consents}

All procedures performed in this study involving human participants were in accordance with the Second Affiliated Hospital of Nanjing Medical University Institutional Ethical Review Board and with the 1964 Helsinki Declaration and its later amendments or comparable ethical standards. Informed consent was obtained from all participants included in the study.

\section{Patients, Donors and FMT Procedure}

FMT via mid-gut for mild to severe $\mathrm{CD}$ with the Harvey Bradshaw Index (HBI) $>4$ as a registered trial was performed from October 2012 to December 2016 at the Second Affiliated Hospital of Nanjing Medical University. This study was retrospectively registered with clinicaltrials.gov. Trial registration date: $13 / 2 / 2013$. Trial registration number: NCT01793831. All patients and donors were informed of the benefits and potential risks of FMT and laboratory screening. All written informed consentd were obtained. Eligible subjects required documentation of definite diagnosis of CD.

Donors were considered to be suitable according to our screening criteria [6]. Healthy donors were selected from patients' relatives or friends or from our universal stool bank (China fmtBank), and carefully screened using the following exclusion criteria: history of drug use (e.g., antibiotic, laxative or diet pill use within the past 3 months; prior immunomodulator or chemotherapy use) and history of disease (e.g., infectious diseases, obesity, diabetes, IBD, irritable bowel syndrome, chronic diarrhea, constipation, colorectal polyps or malignant neoplasm, immunocompromised states, metabolic syndrome, allergy, history of major gastrointestinal operation or autoimmune diseases, as well as any other diseases or conditions related to the disturbance of 
intestinal microbiota). All donors accepted laboratory examinations, such as regular blood tests, C-reactive proteins, erythrocyte sedimentation rates, immunoglobulin subtypes, biochemical tests, hepatitis-associated indices, HIV, syphilis, Cytomegalovirus, Epstein-Barr virus, rubella virus, herpes simplex virus, toxoplasma, and stool testing (including stool culture, stool ova and parasites).

Fecal samples were obtained from scanned donors after signing the informed consent, and were processed for enriching microbiota in the laboratory by manual methods (before April 2014) or automatic methods based on the automatic purification machine GenFMTer (FMT Medical, Nanjing, China) [7] (since April 2014). We followed the "1-h FMT protocol", which means that the time from the stool coming out of the colon to the patient's intestine or storing at $-80^{\circ} \mathrm{C}$ refrigerator is required to be finished within $1 \mathrm{~h}$ [7]. The stored fecal microbiota needed be thawed at $37-39{ }^{\circ} \mathrm{C}$ before infusion into the patient's intestine. However, after we had confirmed that the frozen FMT induced the decreased rate of clinical improvement by $26.7 \%$ at 6 months post-FMT compared with the fresh FMT in CD at our earlier phase [6], the fresh FMT has become the first and even the most important suggestion to patients with CD in our practice. The purified fecal microbiota was delivered into the mid-gut through a naso-jejunal tube or gastroscopic infusion under anesthesia.

\section{The Safety of FMT}

All AEs were recorded during the follow-up after FMT. The longest follow-up time was 5 years. All AEs were described using Common Terminology Criteria for Adverse Events (CTCAE) as in our previous study [6]. Grade refers to the severity of the AE. The CTCAE displays Grades 1-5 with clinical descriptions of severity for AE based on the guideline.

\section{The Efficacy of FMT}

The efficacy of FMT was evaluated at 1 month after FMT. The activity of disease was assessed by HBI based on abdominal symptoms, examination findings, and the presence of extraintestinal manifestations [6, 7]. Clinical response was defined as the HBI score decreasing to $>3$. Clinical remission was defined as HBI $\leq 4$ after FMT. All patients who achieved clinical remission were included in the analysis of clinical response.

\section{Statistical Analysis}

Data were analyzed by using SPSS 18.0. Analyses included the nonparametric test, Chi square test, Fisher's exact test and logistic analysis. Two-tailed $P$ value was calculated with each test. $P<0.05$ was considered significant.

\section{RESULTS}

\section{Patient Characteristics}

A totla of 156 patients with moderate to severe CD underwent FMT from October 2012 to December 2016. Of these, 17 (10.9\%) patients were excluded, as 9 had incomplete medical records, and the remaining 8 patients were lost to follow-up. Finally, 139 patients were included for the analyses (Table 1).

\section{FMT Related AEs}

In total, 139, 106 and 32 patients were included for analysis with 12 months, 2 years and 5 years of follow-up, respectively. All FMTs were delivered through the mid-gut. A total of 33 patients underwent multiple FMTs for maintaining clinical response during a long-term period. The second FMT for CD was performed generally 3-6 months after the first FMT [7]. Six patients were performed the second FMT within 1-3 months after the first FMT in consideration of their serious condition. In total, 184 FMTs were performed for 139 patients. Of these, 13.6\% (25/ 184) of AEs occurred within 1 week after FMT (Fig. 1). Only herpes zoster was observed within 1 week after FMT but it was cured within 1 month. According to CTCAE, the relevance to FMT was classified as probable or possible, and 
Table 1 The baseline characteristics of the included CD patients

\begin{tabular}{ll}
\hline Characteristic & \\
\hline Total patients number, $n$ & 139 \\
Age, year (median) & 32 \\
Sex, male, \% $(n)$ & $61.9 \%(86)$ \\
Age as diagnosis, \% $(n)$ & \\
A1 (age < 16 years) & $15.1 \%(21)$ \\
A2 (age between 17 and 40 years) & $66.2 \%(92)$ \\
A3 (age > 40 year) & $18.7 \%(26)$ \\
Duration of disease, year (median) & 5 \\
Location & \\
L1 (small intestine) & $15.1 \%(21)$ \\
L2 (colonic disease) & $24.5(34)$ \\
L3 (ileocolonic disease) & $59.0(82)$ \\
L4 (upper gastrointestinal tract) & $1.4 \%(2)$ \\
Behavior & \\
B1 (inflammatory) & $45.3(63)$ \\
B2 (stricturing) & $36.0(50)$ \\
B3 (penetrating) & $18.7(26)$ \\
Harvey Bradshaw index (median) & 9 \\
Treatment history & \\
Mesalamines, \% ( $)$ & $95.7(133)$ \\
Steroids, \% ( $n$ ) & $59.7(83)$ \\
Immunosuppressants, \% $(n)$ & $42.4(59)$ \\
Anti-TNF antibody, \% $(n)$ & $21.6(30)$ \\
Surgery, \% ( $n$ ) & $36.0(50)$ \\
Smoking history, \% $(n)$ & $23.7 \%(33)$ \\
\hline
\end{tabular}

84.0\% (21/25) of AE were considered grade 1. All AEs occurred after FMT and $84 \%$ of them were self-improvement without medication treatment (Table 2). Only four patients (16\%) with AEs were treated with medication. One (4\%) had persistent fever after FMT and was prescribed oral prednisone in consideration that the FMT might fail to induce clinical improvement. Hematochezia occurred in one patient after FMT; however, the symptom disappeared after the repeated FMT. One patient required to be discharged due to increased frequency of defecation after FMT. This patient gradually recovered from corticosteroid treatment in another hospital. There were no serious AEs after FMT during the follow-up.

\section{Risk Factors Related to AEs of FMT}

A total of 139 patients at the first FMT were included for further analysis of the possible risk factors for AEs of FMT. Logistic analysis adjusting for the confounders showed that only manual fecal microbiota purification methods were an independent risk factor for FMT-related AEs $\quad(\mathrm{OR}=3.644, \quad 95 \% \quad$ CI $1.414-9.393$, $P=0.007)$. A total of $21.7 \%(15 / 69)$ patients had AEs after FMT in the group of manual methods was lower than the $8.7 \%(10 / 115)$ in the group of automatic preparations (Table 3 ).

\section{The Efficacy of FMT}

We further analyzed the efficacy of FMT and its relevance with the methods of fecal microbiota purification and the occurrence of AEs (Table 4). No significant difference was observed between the group with manual methods and the group with automatic methods, indicating that the method of fecal microbiota preparation should not affect the efficacy of FMT for active CD. Interestingly, the rate of clinical response and clinical remission were $45 \%(9 / 20)$ and $20 \%(4 /$ 20 ) in the patients with $\mathrm{AE}$, which was significantly lower than $75.6 \%(90 / 119)$ and $63.0 \%$ $(75 / 119)$ in the group without $\mathrm{AE}$, respectively.

\section{DISCUSSION}

The present results show that the rate of AEs was $13.6 \%$ for all patients with refractory CD undergoing FMT. Our previously reported data in 2015 based on $30 \mathrm{CD}$ patients [6], and an open label study in 2016 from Boston based on 

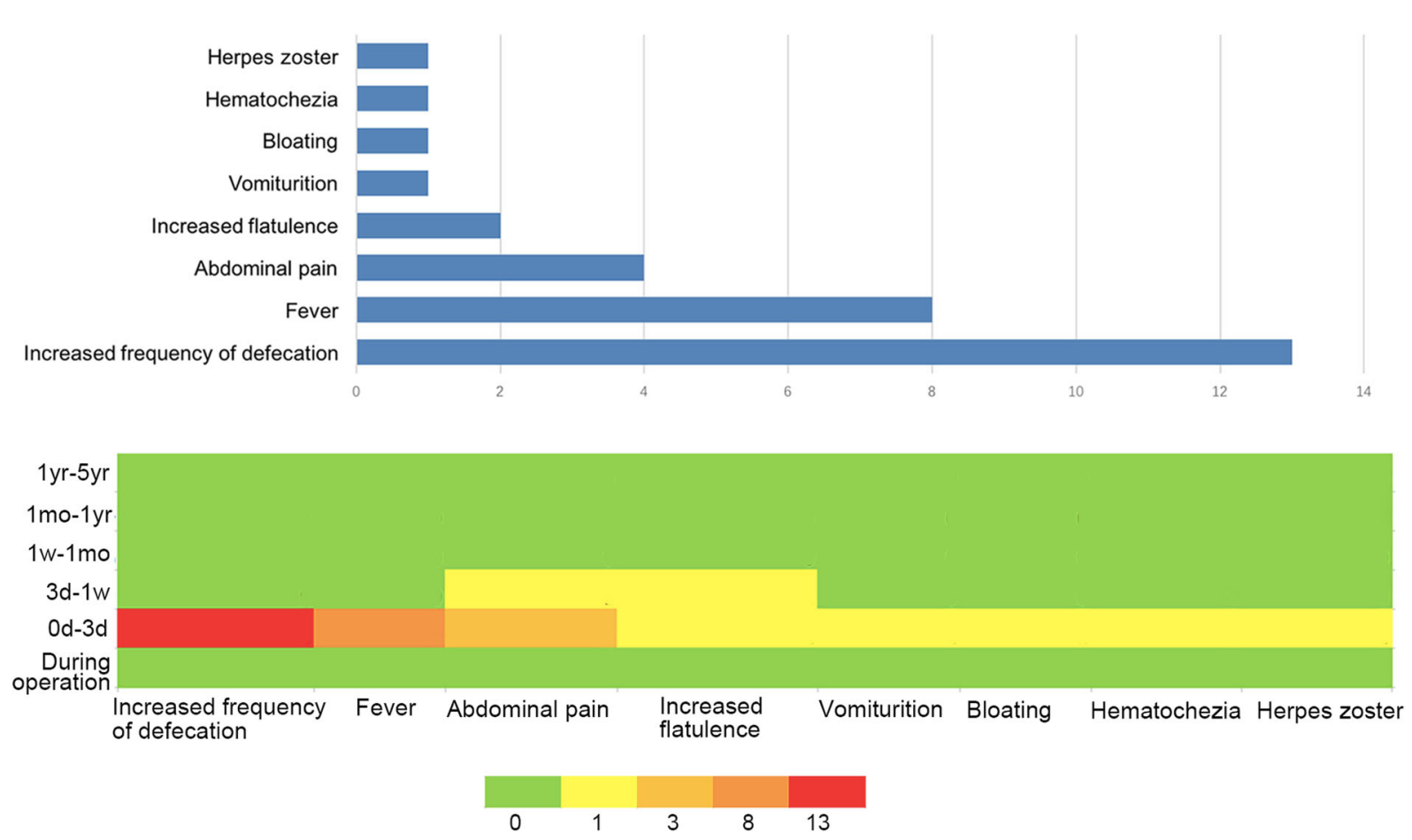

Fig. 1 A total of 184 frequencies of FMT were performed for 139 patients with Crohn's disease. During the longterm follow-up, $13.6 \%(25 / 184)$ of all FMTs had mild AEs, including increased frequency of defecation (13),

19 patients [19], demonstrated a similar overall safety of FMT. A study involving two children from Poland reported that the observed side effects of FMT for CD were self-limiting and benign [20].

One of the most important findings in this study is that the methodology of fecal microbiota preparation in the laboratory is the independent factor related to the occurrence of AEs. In April 2014, our center started to use an automatic machine to enrich the microbiota from the donated healthy stool in a specific laboratory room which was set as GMP level [21]. The following years of clinical experience using FMT indicated that the rate of AEs related to FMT based on automatic purification were significantly less than that related to crude FMT. It should be reasonable that the crude fecal matters may include much more material (e.g., pyrogen) than purificed suspensions from a specifically designed automatic purification system. No AE beyond 1 month was observed. fever (8), abdominal pain (5), flatulence (2), hematochezia (1), vomiturition (1), bloating (1) and herpes zoster (1). All AEs occurred within 1 week after FMT. There were no serious AEs after FMT

Therefore, a 1 month cut-off could be suggested to define short-term and long-term AEs of FMT.

Another question after the purification of microbiota is whether the final materials would decrease the clinical response, since the bacterial components, metabolites, or bacteriophages were observed to play therapeutic roles for CDI [11]. Importantly, we need to note that the automatic purification system was designed to enrich the bacteria instead of collecting moleculars in fecal water. The proper purification process was useful to enrich the microbiota, especially for those bacteria at low kurtosis [6]. Although the present outcome showed a small trend of decreasing clinical efficacy, there was no significant difference in the rate of clinical response or clinical remission between the two groups of different methods. Importantly, the reported clinical response of FMT for CD from different centers was variable $[2-7,19,20,22]$; however, the clinical efficacy in all of our previous studies kept stable $[2,6,7]$. Interestingly, 
Table 2 The details of all adverse events and the relevance to FMT

\begin{tabular}{|c|c|c|c|c|}
\hline Cases & Adverse events & Grade & FMT relevance & $\begin{array}{l}\text { Clinical treatment and } \\
\text { outcome }\end{array}$ \\
\hline & Adverse events rate, $\%(n)$ & & Probable + possible & $13.6 \%(25 / 184)$ \\
\hline 1 & Flatulence & 1 & Probable & Self-improvement \\
\hline 2 & Fever & 2 & Probable & $\begin{array}{l}\text { Improvement after } \\
\text { prednisone use }\end{array}$ \\
\hline 3 & Increased frequency of defecation & 1 & Probable & Self-improvement \\
\hline 4 & Hematochezia & 2 & Possible & $\begin{array}{l}\text { Improvement after repeated } \\
\text { FMT }\end{array}$ \\
\hline 5 & Fever and increased frequency of defecation & 1 & Probable & Self-improvement \\
\hline 6 & Increased frequency of defecation & 3 & Possible & $\begin{array}{l}\text { Improvement after } \\
\text { prednisone use }\end{array}$ \\
\hline 7 & Fever and abdominal pain & 2 & Possible & Self-improvement \\
\hline 8 & Increased frequency of defecation & 1 & Probable & Self-improvement \\
\hline 9 & Increased frequency of defecation & 1 & Probable & Self-improvement \\
\hline 10 & Fever & 1 & Probable & Self-improvement \\
\hline 11 & $\begin{array}{l}\text { Increased frequency of defecation and abdominal } \\
\text { pain }\end{array}$ & 1 & Probable & Self-improvement \\
\hline 12 & $\begin{array}{l}\text { Increased frequency of defecation, flatulence and } \\
\text { abdominal pain }\end{array}$ & 1 & Probable & Self-improvement \\
\hline 13 & $\begin{array}{l}\text { Increased frequency of defecation and abdominal } \\
\text { pain }\end{array}$ & 1 & Probable & Self-improvement \\
\hline 14 & Fever & 1 & Probable & Self-improvement \\
\hline 15 & Fever & 1 & Probable & Self-improvement \\
\hline 16 & Vomiturition & 1 & Possible & Self-improvement \\
\hline 17 & Fever & 1 & Probable & Self-improvement \\
\hline 18 & Fever & 1 & Probable & Self-improvement \\
\hline 19 & Increased frequency of defecation & 1 & Probable & Self-improvement \\
\hline 20 & $\begin{array}{l}\text { Increased frequency of defecation and abdominal } \\
\text { pain }\end{array}$ & 1 & Probable & Self-improvement \\
\hline 21 & Bloating & 1 & Probable & Self-improvement \\
\hline 22 & Increased frequency of defecation & 1 & Probable & Self-improvement \\
\hline 23 & Increased frequency of defecation & 1 & Probable & Self-improvement \\
\hline 24 & Increased frequency of defecation & 1 & Probable & Self-improvement \\
\hline 25 & Herpes zoster & 1 & Possible & Regular treatment \\
\hline
\end{tabular}


Table 3 The risk factors related to adverse events of FMT

\begin{tabular}{|c|c|c|}
\hline Risk factors & Statistics & $P$ value \\
\hline Age & -1.835 & 0.067 \\
\hline Sex & 0.031 & 0.861 \\
\hline Age at diagnosis & -1.748 & 0.080 \\
\hline Duration of disease & -1.123 & 0.261 \\
\hline Location & 1.140 & 0.767 \\
\hline Behavior & 1.560 & 0.459 \\
\hline Harvey Bradshaw index & -0.632 & 0.528 \\
\hline \multicolumn{3}{|l|}{ Treatment history } \\
\hline Mesalamines & 0 & 1 \\
\hline Steroids & 0.920 & 0.338 \\
\hline Immunosuppressants & 0.859 & 0.354 \\
\hline Anti-TNF antibody & 0 & 1 \\
\hline Surgery history & 1.514 & 0.218 \\
\hline Smoking history & 0.756 & 0.385 \\
\hline Family history & 1.173 & 0.279 \\
\hline Total FMT times, $n$ & 184 & \\
\hline Donor source (kinship or non-kinship) & 1.194 & 0.274 \\
\hline Fecal microbiota status (fresh or frozen) & 0 & 0.993 \\
\hline Fecal microbiota purification methods & 6.249 & 0.012 \\
\hline Manual, $\mathrm{AE} \%(n / n)$ & $21.7 \%(15 / 69)$ & \\
\hline Automatic, AE \% $(n / n)$ & $8.7 \%(10 / 115)$ & \\
\hline
\end{tabular}

Table 4 The clinical efficacy related to methods of fecal microbiota preparation and AEs of FMT in CD patients

\begin{tabular}{|c|c|c|c|c|c|c|}
\hline & \multicolumn{3}{|c|}{ Clinical response } & \multicolumn{3}{|c|}{ Clinical remission } \\
\hline & Rate & $x^{2}$ & $P$ & Rate & $x^{2}$ & $P$ \\
\hline \multicolumn{7}{|c|}{ Fecal microbiota preparation methods } \\
\hline Manual & $77.6 \%(52 / 67)$ & 2.576 & 0.108 & $58.2 \%(39 / 67)$ & 0.100 & 0.752 \\
\hline Automatic & $65.3 \%(47 / 72)$ & & & $55.6 \%(40 / 72)$ & & \\
\hline \multicolumn{7}{|c|}{ Adverse events } \\
\hline Yes & $45.0 \%(9 / 20)$ & 7.838 & 0.005 & $20.0 \%(4 / 20)$ & 12.920 & $<0.001$ \\
\hline No & $75.6 \%(90 / 119)$ & & & $63.0 \%(75 / 119)$ & & \\
\hline
\end{tabular}


the present results demonstrated that CD patients with AEs might have decreased benefits from FMT than those patients without AEs. It is valuable to highlight that the risks and benefits were not related to the selected donors according to the current data. However, this does not mean that the screening of donors is not important, because the present results were based on relatively stable donors, a strict FMT protocol, 1-h FMT work flow, and similar quality control in a leading FMT center.

This study had limitations, including nonrandomized design, no microbiota sequencing, no life quality data, and only involving with delivering way through the mid-gut. Another shortcoming was that the activity of disease was assessed by HBI, which has a virtually limited relationship to the $\mathrm{CD}$ endoscopic activity index. Although we have reported the cost-effectiveness analysis of FMT in both CD and ulcerative colitis [23], further study is necessary because of the changed procedures and treatment strategy. The reported evidence of the efficacy and long-term safety of FMT is limited [24]. This is the significance of the national register for 10 years of evaluation of FMT in the USA [25] and the China Microbiota Transplantation System (CMTS) [26]. The present data on safety and efficacy were from CMTS.

\section{CONCLUSION}

The present study highlighted the importance of rethinking the current understanding on the crude FMT process for achieving fecal microbiota suspension. The methodology of FMT is an important factor related to quality control on safety. The present results based on the largest size of CD cases undergoing FMT demonstrated that purification of microbiota from donated healthy stool reduced the rate of AEs, but did not affect the clinical efficacy in the short term.

\section{ACKNOWLEDGEMENTS}

We thank the participants of the study. We appreciate Ms Jie Zhang at CMTS for the professional help.
Funding. This work was supported by publically donated Intestine Initiative Foundation; Jiangsu Province Medicine Creation Team and Leading Talents project; China National Science Foundation (81670495, 81600417); and National Clinical Research Center for Digestive Diseases, Xi'an, China (2015BAI13B07). The article processing charges and Open Access fee were funded by the authors.

Authorship. All named authors meet the International Committee of Medical Journal Editors (ICMJE) criteria for authorship for this article, take responsibility for the integrity of the work as a whole, and have given their approval for this version to be published.

Disclosures. Honggang Wang, Bota Cui, Qianqian Li, Xiao Ding, Pan Li, Ting Zhang, Xiaozhong Yang and Guozhong Ji have nothing to disclose. Faming Zhang is the core inventor of GenFMTer and TET, and the founder of nonprofit China fmtBank.

Compliance with Ethics Guidelines. All procedures performed in studies involving human participants were in accordance with the Second Affiliated Hospital of Nanjing Medical University Institutional Ethical Review Board and with the 1964 Helsinki declaration and its later amendments or comparable ethical standards. Informed consent was obtained from all individual participants included in the study.

Data Availability. The datasets during and/ or analyzed during the current study are available from the corresponding author on reasonable request.

Open Access. This article is distributed under the terms of the Creative Commons Attribution-NonCommercial 4.0 International License (http://creativecommons.org/licenses/ by-nc/4.0/), which permits any noncommercial use, distribution, and reproduction in any medium, provided you give appropriate credit to the original author(s) and the source, provide a link to the Creative Commons license, and indicate if changes were made. 


\section{REFERENCES}

1. Wlodarska M, Kostic AD, Xavier RJ. An integrative view of microbiome-host interactions in inflammatory bowel diseases. Cell Host Microbe. 2015;17:577-91.

2. Zhang FM, Wang HG, Wang $M$, et al. Fecal microbiota transplantation for severe enterocolonic fistulizing Crohn's disease. World J Gastroenterol. 2013;19:7213-6.

3. Suskind DL, Brittnacher MJ, Wahbeh G, et al. Fecal microbial transplant effect on clinical outcomes and fecal microbiome in active Crohn's disease. Inflamm Bowel Dis. 2015;21:556-63.

4. Gordon H, Harbord M. A patient with severe Crohn's colitis responds to Faecal Microbiota Transplantation. J Crohns Colitis. 2014;8:256-7.

5. Kao D, Hotte N, Gillevet P, et al. Fecal microbiota transplantation inducing remission in Crohn's colitis and the associated changes in fecal microbial profile. J Clin Gastroenterol. 2014;48:625-8.

6. Cui B, Feng Q, Wang H, et al. Fecal microbiota transplantation through mid-gut for refractory Crohn's disease: safety, feasibility, and efficacy trial results. J Gastroenterol Hepatol. 2015;30:51-8.

7. He Z, Li P, Zhu J, et al. Multiple fresh fecal microbiota transplants induces and maintains clinical remission in Crohn's disease complicated with inflammatory mass. Sci Rep. 2017;7:4753.

8. Van Nood E, Vrieze A, Nieuwdorp M, et al. Duodenal infusion of donor feces for recurrent Clostridium difficile. N Engl J Med. 2013;368:407-15.

9. Kelly C, Khoruts A, Staley C, et al. Effect of fecal microbiota transplantation on recurrence in multiply recurrent clostridium difficile infection: a randomized trial. Ann Intern Med. 2016;165:609-16.

10. Jiang Z, Ajami NJ, Petrosino JF, et al. Randomised clinical trial: faecal microbiota transplantation for recurrent Clostridum difficile infection-fresh, or frozen, or lyophilised microbiota from a small pool of healthy donors delivered by colonoscopy. Aliment Pharmacol Ther. 2017;45:899-908.

11. Ott SJ, Waetzig GH, Rehman A, et al. Efficacy of sterile fecal filtrate transfer for treating patients with Clostridium difficile infection. Gastroenterology. 2017;152:799-811.

12. Paramsothy S, Kamm MA, Kaakoush NO, et al. Multidonor intensive faecal microbiota transplantation for active ulcerative colitis: a randomised placebo-controlled trial. Lancet. 2017;389:1218-28.

13. Moayyedi P, Surette MG, Kim PT, et al. Fecal microbiota transplantation induces remission in patients with active ulcerative colitis in a randomized controlled trial. Gastroenterology. 2015;149:102-9.

14. Rossen NG, Fuentes $\mathrm{S}$, van der Spek MJ, et al. Findings from a randomized controlled trial of fecal transplantation for patients with ulcerative colitis. Gastroenterology. 2015;149:110-8.

15. Uygun A, Ozturk K, Demirci H, et al. Fecal microbiota transplantation is a rescue treatment modality for refractory ulcerative colitis. Medicine. 2017;96:e6479.

16. Angelberger S, Reinisch W, Makristathis A, et al. Temporal bacterial community dynamics vary among ulcerative colitis patients after fecal microbiota transplantation. Am J Gastroenterol. 2013;108:1620-30.

17. Hoffmann D, Palumbo F, Ravel J, et al. Improving regulation of microbiota transplants. Science. 2017;358:1390-1.

18. Kelly CR, Ihunnah $C$, Fischer $M$, et al. Fecal microbiota transplant for treatment of Clostridium difficile infection in immunocompromised patients. Am J Gastroenterol. 2014;109:1065-71.

19. Vaughn BP, Vatanen T, Allegretti JR, et al. Increased intestinal microbial diversity following fecal microbiota transplant for active Crohn's disease. Inflamm Bowel Dis. 2016;22:2182-90.

20. Karolewska-Bochenek K, Grzesiowski P, Banaszkiewicz A, et al. A two-week fecal microbiota transplantation course in pediatric patients with inflammatory bowel disease. Adv Exp Med Biol. 2018;1047:81-7.

21. Cui B, Li P, Xu L, et al. Step-up fecal microbiota transplantation (FMT) strategy. Gut Microbes. 2016;7:323-8.

22. Bak SH, Choi HH, Lee J, et al. Fecal microbiota transplantation for refractory Crohn's disease. Intest Res. 2017;15:244-8.

23. Zhang T, Xiang J, Cui B, et al. Cost-effectiveness analysis of fecal microbiota transplantation for inflammatory bowel disease. Oncotarget. 2017;8:88894-903.

24. Jeon SR, Chai J, Kim C, et al. Current evidence for the management of inflammatory bowel diseases 
using fecal microbiota transplantation. Curr Infect Dis Rep. 2018;20:21.

25. Kelly CR, Kim AM, Laine L, et al. The AGA's fecal microbiota transplantation national registry: an important step toward understanding risks and benefits of microbiota therapeutics. Gastroenterology. 2017;152:681-4.

26. Zhang F, Cui B, He X, et al. Microbiota transplantation: concept, methodology and strategy for its modernization. Protein Cell. 2018;9:462-73. 